\title{
A child with familial glomerulonephritis: Questions
}

\section{Marco Pennesi ${ }^{1}$ (D) . Domenica Squillaci ${ }^{2} \cdot$ Francesca Diomedi-Camassei $^{3} \cdot$ Giulia Pennesi $^{4} \cdot$ Egidio Barbi $^{1,2}$}

Received: 19 March 2020 / Accepted: 23 March 2020 / Published online: 14 April 2020

(C) IPNA 2020

A 24-month-old boy was referred for a glomerular hematuria previously labeled as a chronic glomerulonephritis.

His clinical story begins at the age of 18 months when the urine test performed due to the presence of dysuria showed the presence of microhematuria and light proteinuria. Farley's test results highlighted glomerular hematuria. Blood tests performed in the same occasion were all normal as well as kidneys and urinary tract ultrasound.

Family history was remarkable due to the mother's diagnosis of a mesangial proliferative glomerulonephritis without hearing loss or eye anomalies at 3 years of age, currently treated with losartan. Maternal grandfather was reported to have suffered from a non-defined form of chronic renal failure.

Blood laboratory test results were all in normal range, and no anomalies were detected at the renal ultrasound. Both the eye and the ENT examination gave normal results. The result of the genetic test for Alport syndrome (COL4A3, COL4A4, COL4A5) was negative.

\section{Questions}

1. What is the most likely diagnosis?

2. Which test(s) would be required to confirm the suspected diagnosis?

3. What is the prognosis and treatment of the disease?

\section{Compliance with ethical standards}

Conflict of interest The authors declare that they have no conflict of interest.

Publisher's note Springer Nature remains neutral with regard to jurisdictional claims in published maps and institutional affiliations.

The answers to these questions can be found at https://doi.org/10.1007/ s00467-020-04551-1.

Marco Pennesi

marco.pennesi@burlo.trieste.it

Institute for Maternal and Child Health - IRCCS Burlo Garofolo, Trieste, Italy

2 University of Trieste, Trieste, Italy

3 Bambino Gesù Children's Hospital, IRCCS, Rome, Italy

4 Edinburgh Napier University, Edinburgh, UK 